ORIGINAL ARTICLE

\title{
Prevalence of Malnutrition and Associated Factors among Hospitalized Patients with Acquired Immunodeficiency Syndrome in Jimma University Specialized Hospital, Ethiopia
}

\author{
Habtamu Mulu ${ }^{1}$, Leja Hamza ${ }^{1}$, Fesehaye Alemseged ${ }^{2}$
}

\begin{abstract}
BACKGROUND: HIVIAIDS predisposes to malnutrition. Malnutrition exacerbates HIV/AIDS progression resulting in increased morbidity and mortality. The magnitude of malnutrition in HIV/AIDS patients has not been well studied in Ethiopian setup. Our objective was to assess the prevalence of malnutrition and associated factors among HIV/AIDS patients admitted to Jimma University Specialized Hospital (JUSH).

METHOD: A cross-sectional study was conducted to assess the nutritional status of 109 HIV/AIDS patients admitted from November 2013 to July 2014. Cohort design was also used for outcome assessment. Serum levels of hemoglobin, albumin and CD4 counts were determined. Data were organized, coded, cleaned, entered into a computer and analyzed using SPSS version 16.0. Descriptive analysis was done initially. Those variables in the bivariate analysis with $P$-value $<0.25$ were then considered as candidates to be included in the multivariable logistic regression model. A P-vale of $<0.05$ was considered as statistically significant.

RESULTS: The mean age of the patients was 32.7 \pm 8.12 with male to female ratio of 1:1.9. Patients were in either clinical stage, 3(46.8\%), or stage, 4(53.2\%). Forty nine (45\%) of the respondents had a CD4 count of $<200$ cells $/ \mu L$. The overall prevalence of malnutrition was $46.8 \%(B M I<18.5 \mathrm{~kg} / \mathrm{m} 2)$ and $44.1 \%$ (MUAC $\leq 20 \mathrm{~cm})$. Eighty four (77.1\%) of the patients had a serum albumin level of $\leq 3.5 \mathrm{~g} / \mathrm{dl}$ while 76 (69.6\%) of the patients had anemia $(\mathrm{Hg}<12 \mathrm{~g} / \mathrm{dl})$.

CONCLUSION: The prevalence of malnutrition was found to be high. WHO Stage 4 disease and CD4

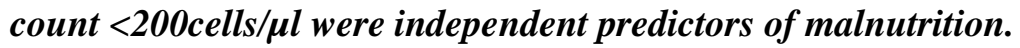

KEYWORDS: Malnutrition, HIVIAIDS, WHO Clinical Stage, CD4 count, Jimma, Ethiopia
\end{abstract}

DOI: http://dx.doi.org/10.4314/ejhs.v26i3.4

\section{INTRODUCTION}

Malnutrition is defined as the cellular imbalance between supply of nutrients and energy and the body's demand for them to ensure growth, maintenance and specific functions $(1,2)$. Nutrition and HIV are strongly related and complement each other. Both can independently cause progressive damage to the immune system. Malnutrition is a frequent problem in persons infected with HIV. It adds fuel to the fire by accelerating the progress of HIV infection to
AIDS. HIV/AIDS is also associated with biological and social factors that affect the individual's ability to consume, utilize and acquire food $(1,3)$.

Causes of malnutrition in patients with AIDS may be multi-factorial. It includes disorders of food intake, opportunistic infections, diarrhea, nutrient malabsorption and wasting syndrome $(3,4,5,6)$. Furthermore, HIV specifically affects nutritional status by increasing energy requirements through alterations in metabolism

\footnotetext{
${ }^{1}$ Department of Internal Medicine, College of Health Sciences, Jimma University, Ethiopia.

${ }^{2}$ Department of Epidemiology and Biostatistics, College of Health Sciences, Jimma University, Ethiopia.

Corresponding Author: Leja Hamza, Email: lejahamza@yahoo.com
} 
and oxidative stress $(5,6,7)$. HIV/AIDS patients require $10 \%$ more energy when asymptomatic and 20-30\% more when symptomatic compared to uninfected individuals (4).

The world's highest HIV infection rates are found in Sub-Saharan Africa (SSA). Food shortages and malnutrition have combined with HIV/AIDS to bring some countries to the brink of crisis $(8,9)$. Malnutrition and food insecurity are associated with increased mortality and poor clinical outcomes among people living with HIV/AIDS (10). The HIV/AIDS epidemic has had security and overall socioeconomic development in countries that have been greatly affected by the disease (11).

Attention to the problems of malnutrition in patients with AIDS is of paramount importance because the timing of death in these patients may be more closely related to the degree of body cell mass depletion than to any specific underlying infection [3]. The nutritional problems have been shown to be significant and contribute to morbidity and mortality in HIV+/AIDS patients (8). Evidence indicates that even relatively small losses in weight $(5 \%)$ are associated with decreased survival rate (12). There is an urgent need for renewed focus on and use of resources for nutrition as a fundamental part of the comprehensive package of care at country level (7, 11).

It has been postulated that the use of HAART would reduce the occurrence of HIV associated weight loss and wasting (13). Commencement of HAART in HIV infected individuals exhibits benefits to the circulating serum albumin levels (14).

Malabsorption resulting from intestinal problems leads to subtherapeutic levels of ARV drugs and treatment failure. Thus, patients with malabsorption could require dose adjustment and/or dietary supplements during periods of diarrheal illness (5).

Malnutrition at the time of starting ART was significantly associated with decreased survival $(12,15,16)$. Low BMI at ART initiation was associated with poor treatment outcomes. Conversely, normal BMI was associated with adequate CD4 response and reduced lost to follow up cases (17). BMI at diagnosis is a strong, independent predictor of survival in HIV-infected patients in West Africa. In the absence of sophisticated clinical and laboratory support, BMI may also prove a useful guide for deciding when to initiate antiretroviral therapy $(10,18)$.

Unemployment, WHO clinical AIDS stage four, one or more number of previous opportunistic infections and gastrointestinal symptoms were found to be important risk factors for malnutrition among people living with HIV/AIDS (6). Tuberculosis, central nervous system toxoplasmosis and sepsis were the three most common disease complexes among admissions and deaths (19).

Anemia is the most common hematologic abnormality in HIV patients. Anemia is a predictor of progression from HIV infection to AIDS or death; more than $70 \%$ of HIV patients develop anemia and thus require blood transfusion (20). Anemia, thrombocytopenia and severe malnutrition were strong independent predictors of mortality (16).

Literatures on the prevalence and factors associated with malnutrition among people living with HIV/AIDS are limited in Ethiopia and not well documented either (6). Only few studies were done on malnutrition and HIV/AIDS in Ethiopia $(6,7,19,20)$. There are no such studies done in hospitalized HIV/AIDS patients in Jimma. Therefore, the aim of this study was to assess the prevalence of malnutrition and associated factors in HIV/AIDS patients who were admitted to medical wards of our hospital during the study period. The outcome of this study is expected to encourage healthcare providers to strengthen the quality of nutritional assessment and care. It can also serve as a baseline for further large scale studies on nutrition and HIV/AIDS.

\section{METHODS AND PATIENTS}

The study was done on HIV/AIDS patients hospitalized to Jimma University Specialized Hospital located in Jimma Town, Southwest Ethiopia. The hospital serves as a teaching and referral hospital with a catchment of 15 million people. The hospital has three medical wards with 85 admission beds. It also has a chronic care and training centre for HIV/AIDS and TB patients.

A hospital based prospective cross-sectional study design was used to study all adult ( $\geq 18$ yrs of age) HIV/AIDS patients admitted to medical wards from November 10, 2013 to July 10, 2014. We also used cohort design to assess our secondary 
objective, i.e. the outcome of patients with malnutrition at the end of discharge or at the end of the study. The required sample size was calculated using single population proportion formula, assuming the prevalence of malnutrition among HIV/AIDS patients to be $50 \%$. It was calculated to be 384 . From the hospital record, the total number of HIV/AIDS patients admitted to medical wards in the preceding year was 161 . Applying a formula for finite population correction, the final required sample size to conduct the study was 114 . To get the required sample size, we used convenience sampling. Therefore, All HIV/AIDS patients admitted to JUSH medical wards over the study period were included in the study. Patients with cognitive impairment and those who required immediate intensive care support were excluded. Accordingly, out of 114 admitted HIV/AIDS patients who were admitted during the study period, 109 patients who fulfilled the inclusion criteria were included in the study.

Data were collected by trained medical residents working in medical wards during the study period. Ethical clearance was obtained from the Ethical Review Board of the College of Public Health and Medical Sciences, Jimma University College. The medical director, ward physicians and nurses were contacted before starting data collection. The aim of the study was explained to the study participants. They understood that it has no any harm on them. No one was deprived of any medical treatment and all malnourished individuals were treated in accordance with the protocol of the hospital. To maintain confidentiality of the study participants, any personally identifiable information (e.g. name, address) was not recorded on the data collection format.

A structured questionnaire was used to gather sociodemographic information, and relevant clinical history was collected by interviewing patients using a structured questionnaire. Main variables of interest were time since HIV diagnosis, prior HIV related hospitalizations, medical conditions related to current hospitalization, duration of hospital stay, HAART status, history of drug interruption [adherence] and patient response to therapy [outcome]. Adherence to HAART was defined as regular use of ARVs at the right frequency of dosing and timing. Ability to keep to this pattern of utilization was defined as $100 \%$ adherence, while adherence of $>95 \%$ was accepted as optimal adherence. Levels of adherence below 95\% were considered to be sub-optimal.

Nutritional status was assessed using Body Mass Index [BMI], Mid Upper Arm Circumference (MUAC), serum Albumin and serum Hemoglobin levels. Patients with $\mathrm{BMI}<18.5 \mathrm{~kg} / \mathrm{m} 2$, MUAC $<20 \mathrm{~cm}$ or serum albumin $<3.5 \mathrm{~g} / \mathrm{dl}$ were categorized as malnourished $(3,4,14,18)$. Patients were systematically evaluated for presence of opportunistic diseases and co-morbid illnesses which were further supplemented with the data obtained through patient interview and chart review. CD4 count was determined at the time of admission. Clinical outcome [i.e. patients' clinical status or response to therapy] was assessed at the end of the study period or in-between if an event like discharge, ICU transfer, or death occurred. The occurrence of death, prolonged hospitalization [ $\geq 14$ days], need of ICU admission were considered as unfavorable outcomes. Anthropometric and laboratory measurements were done in a standard way. Weight was measured with a weight scale and height was measured with a height scale after daily calibration before starting data collection. The mean of three measurements of each of the anthropometric measurements were taken and reported as the final measurement. For laboratory assessments of nutritional status, blood tests for serum albumin and hemoglobin were performed by an experienced laboratory technologist. A $5 \mathrm{ml}$ blood sample was taken from the cubital fossa, 2.5 $\mathrm{ml}$ was put in an EDTA tube for complete blood count and $2.5 \mathrm{ml}$ was put in a tube with no anticoagulants for serum albumin determination. Serum albumin was determined by HORIBA medical $^{\mathrm{TM}}$ ABX Pentra 400 with the use of appropriate human serum controls. Complete blood count determination was done using Cysmex KX21 machine. All results were expressed to the nearest 0.1 decimal place. The data collection instrument was pre-tested on $5 \%$ of the total sample size before actual data collection. The process of data collection was daily supervised and monitored by the principal investigator. The completeness and consistency of data is checked before compilation. The compiled 
data were organized, cleaned, coded, entered into a computer and analyzed using SPSS version 16.0. First, descriptive analysis was done. Then, bivariate analysis was done to identify factors associated with nutritional status. Those variables with $\mathrm{P}-$ value $<0.25$ in the bivariate analysis were considered as candidates to be included in the multivariable logistic regression model. A P-vale of $<0.05$ was considered as statistically significant.

\section{RESULTS}

Socio-demographic characteristics: A total of 114 HIV/AIDS patients were admitted to medical wards during the study period. One hundred and nine of them fulfilled the inclusion criteria and included in the analysis. Two patients with cognitive impairment, another two patients a devastating impact on health, nutrition, food who were directly admitted to ICU, and one patient who refused to be included in the study were excluded from the study. This resulted in a response rate of $95.6 \%$.

Seventy-two $(66.1 \%)$ of the patients were females. The mean age of the study subjects was $32.7 \pm 8.12$. Fifty $(45.9 \%)$ of them were in the age group of 25-34 years. Only $20(18.5 \%)$ of the respondents had completed college level education. Thirty-four $(31.2 \%)$ were farmers. The majority of the study subjects, 68(62.2\%), were urban residents. The majority of the patients, $83(76.1 \%)$, had poor socioeconomic status with annual income of $<1700$ Birr, and the mean monthly income was 978 Birr with the majority being in the $2^{\text {nd }}$ and $3^{\text {rd }}$ quartiles (Table 1 ).

Table 1: Socio-demographic characteristics of HIV/AIDS patients admitted to JUSH from November 2013 to July 2014.

\begin{tabular}{llll}
\hline $\begin{array}{l}\text { Sociodemographic } \\
\text { Characteristics }\end{array}$ & Category & Frequency & Percent \\
\hline Age & $<24$ & 11 & 10.1 \\
& $25-34$ & 50 & 45.9 \\
& $35-44$ & 37 & 33.9 \\
Sex & $\geq 45$ & 11 & 10.1 \\
& Male & 37 & 33.9 \\
Educational status & Female & 72 & 66.1 \\
& Illiterate & 39 & 35.8 \\
& Primary education & 30 & 27.5 \\
& Secondary education & 20 & 18.5 \\
Occupation & College and above & 20 & 18.5 \\
& Government employee & 28 & 25.5 \\
& Merchant & 11 & 10.1 \\
& Farmer & 34 & 31.2 \\
Monthly income & Student & 6 & 5.5 \\
(in Birr) & unemployed & 30 & 27.5 \\
& $<193$ & 27 & 24.8 \\
& $193-420$ & 28 & 25.7 \\
Residence & $421-1700$ & 28 & 25.7 \\
& $>1700$ & 26 & 23.9 \\
& Urban & 68 & 62.4 \\
\hline
\end{tabular}

Clinical characteristics: Seventy five $(68.8 \%)$ of the study subjects were known HIV/AIDS patients already on chronic care and follow-up. Of these, $64(85 \%)$ were on HAART. Fifteen $(23.4 \%)$ of the patients taking HAART had sub-optimal $(<95 \%)$ adherence. All of the patients admitted were in either WHO clinical stage III, 51(46.8\%) and stage IV, 58(53.2\%). The average current 
CD4 count was $247.7 \pm 165$ cells/liter, and $49(45 \%)$ of the respondents had a CD4 count of less than 200 cells/liter. The major medical conditions associated with the current hospitalization were tuberculosis (both pulmonary and disseminated TB), 36(33\%), bacterial pneumonia, 24(22\%), severe anemia, 24(22\%), and oro-esophageal candidiasis $18(16.5 \%)$. The average hospital stay was $19.3 \pm 10.6$ days and $69(63.3 \%)$ of the patients were hospitalized for more than 14 days. Patients with malnutrition stayed for 22.7 days on average, while those without malnutrition stayed for 16.3 days. A large proportion of patients with malnutrition $(80.3 \%, n=41)$ had greater than 14 days of hospital stay as compared to those who are not malnourished (48.3\%, $\mathrm{n}=28)$. Most, $70(64.2 \%)$, of the patients were discharged improved, while $23(21.1 \%)$ of them died (Table 2).

Table 2: Clinical characteristics of HIV/AIDS patients admitted to JUSH from November 2013 to July 2014

\begin{tabular}{llll}
\hline Clinical Characteristics & Category & Frequency & Percentage \\
\hline Time since HIV Diagnosis & New & 34 & 31.2 \\
& $<2$ years & 33 & 30.3 \\
On HAART & $>2$ years & 42 & 38.5 \\
& Yes & 64 & 58.7 \\
Medical conditions associated with current & No & 45 & 41.3 \\
hospitalization & Tuberculosis & 36 & 33 \\
& Pneumonia & 24 & 22 \\
& Severe anemia & 24 & 22 \\
& PCP & 8 & 7.3 \\
& Oro-esophageal candidiasis & 18 & 16.5 \\
& Bacterial meningitis & 9 & 8.3 \\
& CNS Toxoplasmosis & 6 & 5.5 \\
& Cryptococcal meningitis & 4 & 3.7 \\
& Chronic diarrhea & 7 & 6.4 \\
& GI onset sepsis & 4 & 3.7 \\
WHO Clinical Stage & CLD, chronic viral & 4 & 3.7 \\
& hepatitis & 18 & 16.5 \\
CD4 Count at admission & Others* & 40 & 36.7 \\
Days of hospitalization & 2 or more comorbidities & & \\
& Stage III & 51 & 46.8 \\
Outcome & Stage IV & 58 & 53.2 \\
& $<200$ & 49 & 45 \\
& $\geq 200$ & 60 & 55 \\
& $<14$ days & 40 & 36.7 \\
& $\geq 14$ days & 69 & 63.3 \\
& Improved & 70 & 64.2 \\
& The Same & 7 & 6.4 \\
& ICU admission & 9 & 8.3 \\
& Death & 23 & 21.1 \\
\hline
\end{tabular}

*NHL, acute viral hepatitis, IRIS, brain abcess, Kaposi sarcoma, splenic abcess, ESRD

Nutritional status: The average BMI and MUAC was $19.0 \pm 3.39$ and $21.5 \pm 3.75$, respectively. Fiftyone $(46.8 \%)$ and $46(42.2 \%)$ of the patients were malnourished using BMI $<18.5 \mathrm{~kg} / \mathrm{m} 2$ and MUAC $<20 \mathrm{~cm}$, respectively. The mean serum albumin was $2.87+0.73$. Eighty-four $(77.1 \%)$ of the patients had a serum albumin of less than $3.5 \mathrm{~g} / \mathrm{liter}$. The mean hemoglobin was $9.7 \pm 3$. Seventy-six $(69.6 \%)$ of the patients were anemic $(\mathrm{Hg}<12 \mathrm{~g} / \mathrm{dl})$, and $19(17.7 \%)$ of them had severe anemia (Table 3 ). 
Table 3: Nutritional status of HIV/AIDS patients admitted to JUSH from November 2013 to July 2014.

\begin{tabular}{llll}
\hline $\begin{array}{l}\text { Nutritional } \\
\text { status }\end{array}$ & Category & Frequency & Percent \\
\hline Body mass & $<18.5$ & 51 & 46.8 \\
index & $\geq 18.5$ & 58 & 53.2 \\
MUAC & $<20$ & 46 & 42.2 \\
& $\geq 20$ & 63 & 57.8 \\
Albumin & $<3.5$ & 84 & 77.1 \\
& $\geq 3.5$ & 25 & 22.9 \\
Hemoglobin & $<7$ & 27 & 24.8 \\
& $7-9.9$ & 30 & 27.5 \\
& $10-11.9$ & 19 & 17.4 \\
& $\geq 12$ & 33 & 30.3 \\
\hline
\end{tabular}

Factors associated with malnutrition: Among the socio-demographic variables, only female sex and being farmer were found to have statistical association with malnutrition (Table 4).

The clinical variables significantly associated with malnutrition were longer duration (> 2years) since HIV diagnosis, having two or more opportunistic diseases/co-morbidities, longer duration ( $>14$ days) of hospital stay, not being on HAART, suboptimal adherence to HAART, advanced clinical stage and in-hospital mortality. Similarly, CD4 count $<200$ cell $/ \mu 1$, serum albumin <3.5 gm/dl and serum hemoglobin < $7 \mathrm{gm} / \mathrm{dl}$ were found to be significantly associated with malnutrition (Table 5).

Table 4: Association of socio-demographic variables with nutritional status in HIV/AIDS patients admitted to JUSH, from November 2013 to July 2014.

\begin{tabular}{|c|c|c|c|c|}
\hline \multirow[t]{2}{*}{ Variables } & \multirow[t]{2}{*}{ Category } & \multicolumn{2}{|c|}{ Nutritional status } & \multirow[b]{2}{*}{ P-value } \\
\hline & & $\mathrm{BMI} \geq 18.5$ & $\mathrm{BMI}<18.5$ & \\
\hline \multirow[t]{2}{*}{ Sex } & Male & 20 & 17 & \multirow{2}{*}{$\begin{array}{l}0.016 \\
0.899\end{array}$} \\
\hline & Female & 38 & 34 & \\
\hline \multirow[t]{4}{*}{ Age group } & $\leq 24$ & 5 & 6 & \multirow{4}{*}{$\begin{array}{l}1.863 \\
0.601\end{array}$} \\
\hline & $25-34$ & 28 & 22 & \\
\hline & $35-44$ & 21 & 16 & \\
\hline & $>44$ & 4 & 7 & \\
\hline \multirow[t]{4}{*}{ Marital status } & Single & 9 & 12 & \multirow{4}{*}{$\begin{array}{l}3.099 \\
0.377\end{array}$} \\
\hline & Married & 28 & 18 & \\
\hline & Divorced & 9 & 6 & \\
\hline & Widowed & 12 & 15 & \\
\hline \multirow[t]{2}{*}{ Residency } & Urban & 40 & 28 & \multirow{2}{*}{$\begin{array}{l}2.287 \\
0.130\end{array}$} \\
\hline & Rural & 18 & 23 & \\
\hline \multirow{4}{*}{$\begin{array}{l}\text { Educational } \\
\text { status }\end{array}$} & Illiterate & 19 & 20 & \multirow{4}{*}{$\begin{array}{l}7.340 \\
0.62\end{array}$} \\
\hline & Primary & 13 & 17 & \\
\hline & Secondary & 10 & 10 & \\
\hline & College \& above & 16 & 4 & \\
\hline \multirow[t]{5}{*}{ Occupation } & Government employee & 22 & 6 & \multirow{5}{*}{$\begin{array}{l}13.258 \\
0.021\end{array}$} \\
\hline & Merchant & 4 & 7 & \\
\hline & Student & 3 & 3 & \\
\hline & Farmer & 16 & 18 & \\
\hline & Unemployed & 13 & 17 & \\
\hline \multirow[t]{4}{*}{ Income (in birr) } & <193birr/month & 12 & 15 & \multirow{4}{*}{$\begin{array}{l}4.143 \\
0.246\end{array}$} \\
\hline & 193-420birr/month & 12 & 16 & \\
\hline & 421-1700birr/month & 18 & 10 & \\
\hline & >1700birr/month & 16 & 10 & \\
\hline
\end{tabular}


Table 5: Association clinical and laboratory variables with nutritional status in HIV/AIDS patients admitted to JUSH, from November 2013 to July 2014.

\begin{tabular}{|c|c|c|c|c|}
\hline \multirow[b]{2}{*}{ Variables } & \multirow[b]{2}{*}{ Category } & \multicolumn{2}{|c|}{ Nutritional status } & \multirow[b]{2}{*}{ P-value } \\
\hline & & BMI $>18.5$ & BMI $<18.5$ & \\
\hline \multirow[t]{3}{*}{ Time of HIV diagnosis } & New & 12 & 22 & 6.384 \\
\hline & $<2$ years & 20 & 13 & 0.041 \\
\hline & $\geq 2$ years & 26 & 16 & \\
\hline \multirow[t]{2}{*}{2 or more co-morbidities } & Yes & 9 & 31 & 23.938 \\
\hline & No & 49 & 20 & 0.001 \\
\hline \multirow{2}{*}{ WHO Clinical stage } & Stage 3 & 40 & 11 & 24.486 \\
\hline & Stage 4 & 18 & 40 & 0.000 \\
\hline \multirow[t]{2}{*}{ HAART } & Yes & 40 & 24 & 5.373 \\
\hline & No & 18 & 27 & 0.020 \\
\hline \multirow[t]{2}{*}{ HAART Adherence } & Optimal & 34 & 15 & 4.232 \\
\hline & Sub-optimal & 6 & 9 & 0.040 \\
\hline \multirow[t]{2}{*}{ Prior HIV related admission } & Yes & 19 & 17 & 2.137 \\
\hline & No & 27 & 12 & 0.144 \\
\hline \multirow[t]{2}{*}{ CD4 count at admission } & $<200$ & 18 & 31 & 9.706 \\
\hline & $\geq 200$ & 40 & 20 & 0.002 \\
\hline \multirow[t]{2}{*}{ Mortality } & Yes & 8 & 15 & 3.976 \\
\hline & No & 50 & 36 & 0.046 \\
\hline \multirow[t]{2}{*}{ Hospital stay } & $<14$ days & 30 & 10 & 12.049 \\
\hline & $\geq 14$ days & 28 & 41 & 0.001 \\
\hline \multirow[t]{2}{*}{ Serum albumin } & $<3.5$ & 35 & 49 & 19.605 \\
\hline & $\geq 3.5$ & 23 & 2 & 0.001 \\
\hline \multirow[t]{4}{*}{ Hemoglobin } & $\overline{<7}$ & 9 & 18 & 17.009 \\
\hline & $7-9.9$ & 11 & 19 & 0.001 \\
\hline & $10-11.9$ & 12 & 7 & \\
\hline & $\geq 12$ & 26 & 7 & \\
\hline
\end{tabular}

However, multiple logistic regression analysis showed that only advanced clinical disease and lower CD4 count were independent predictors of malnutrition. Patients with stage 4 disease had 14.5 times more likelihood of being malnourished
(95\% CI 3.621, 58.297). Similarly, the likelihood of malnutrition in patients with baseline CD4 count $<200$ cells $/ \mu$ liter was 5.7 times more than their counterparts $(95 \%$ CI 1.286, 25.375) (Table $6)$. 
Table 6: Multiple logistic regressions of clinical stage and baseline CD4 count with nutritional status of HIV/AIDS patients admitted to JUSH, from November 2013 to July 2014.

\begin{tabular}{llllll}
\hline Variables & Category & Nutritional status & p-value & AOR(95.0\% CI) \\
\cline { 3 - 4 } & & BMI(18.525.0)(Ref) & BMI $(<18.5)(1)$ & & \\
\hline Clinical & Stage 3 & 40 & 11 & .000 & 1 \\
stage & Stage 4 & 18 & 40 & & $14.529(3.621,58.297)$ \\
CD4 & $<200$ & 18 & 31 & .022 & 1 \\
count at & $>200$ & 40 & 20 & .000 & .045 \\
admission & Constant & & & & \\
\hline
\end{tabular}

\section{DISCUSSION}

A total of 109 HIV/AIDS patients were included in the analysis. Fifty (45.9\%) of the study subjects were in the age group of 25-34 years, which is the most affected age group by HIV according to Ethiopian Demographic and Health survey [DHS] 2011 report. This is also in line with findings from other studies in similar setups $(4,6,19)$.

The overall prevalence of malnutrition was found to be high. This is comparable with the rate of malnutrition in hospitalized AIDS patients in similar setups $(6,16,17,19,20)$. This is because most of the admitted AIDS patients had advanced disease stage with CD4 counts $<200 / \mu \mathrm{L}$ which is known to be related with malnutrition. The higher rate of concomitant opportunistic diseases like tuberculosis in such patients could also contribute to the high prevalence of malnutrition. Similarly, most of our patients were also found to be anemic. This can be justified by the advanced stage of the disease, high prevalence of malnutrition, low socioeconomic status, high occurrence of co-morbidities and drug side effects $(3,6,7,10,16,19)$.

All of the patients admitted were either WHO clinical stage III or stage IV. Thirty nine $(35.8 \%)$ of our patients had unfavorable outcome with overall mortality of $21.1 \%$. The major medical condition associated with current hospitalization was tuberculosis $(33 \%)$ followed by pneumonia $(22 \%)$ and anemia (22\%). Having two or more comorbid medical conditions at admission had a significant association with malnutrition ( $p$ value $=0.001$ ). Similarly, long duration of hospital stay and in-hospital death were statistically associated with malnutrition ( $\mathrm{p}$ value $=0.019 ; \mathrm{p}$ value $=0.007$, respectively). This is also in line with the commonest causes of HIV/AIDS related admissions and mortality worldwide $(6,7,9,10,12,16,17,18,19)$. Patients with clinical stage 4 disease were found to have more than 14 times higher likely hood of being malnourished compared to those with clinical stage 3 disease ( $\mathrm{AOR}=14.529 ; 95.0 \% \mathrm{CI}=3.621,58.297)$. This is also comparable with other studies which showed similar results $(3,6,7,12,15,16,19)$.

Most of the patients were hospitalized for 14 or more days. The length of hospital stay had a statistically significant association with nutritional status $(\mathrm{P}=0.001)$. The average hospital stay was $19.3 \pm 10.6$ days. This is a shorter duration compared to the mean length of hospital stay (27.5 days) found by a similar study done in a national referral hospital (19). Since the later study was conducted in a national referral hospital, there is increased chance of admitted patients being more complicated and critical who are referred from all regions of the country. Moreover, it was conducted in 2003 before the wide spread use of HAART which is important for favourable treatment outcome and shorter hospital stay. On the other hand, the duration of hospital stay in our study is longer than the average HIV-related hospital stays in Australia (14 days) and in USA (6.9 days). This could be due to the fact that the studies were done at different setups and level of standards of care. The higher rate of malnutrition could also contribute to the prolonged hospital stay in our study. Other similar studies done in Ethiopian setup did not analyze the length of hospital stay $(6,7,20)$.

Similarly, patients with CD4 count below 200 cells/uliter had more than fivefolds chance of being malnourished compared to those with CD4 count $\geq 200 \mathrm{cells} / \mu$ liter $\quad(\mathrm{AOR}=5.713 ; \quad 95.0 \%$ $\mathrm{CI}=1.286,25.375)$. Similar studies done in 
Ethiopian setup $(6,7,20)$ showed similar association between CD4 count and nutritional status. The finding also goes with similar studies done in other developing countries $(4,16,17)$. Even a study done in developed countries showed statistically significant association of lower CD4 count $(<100 \mathrm{cells} / \mu \mathrm{l})$ with malnutrition (14).

In conclusion, most of our patients were younger with male to female ratio of 1:1.9. The majority of them were also found to have poor socioeconomic status and advanced stage of AIDS with high rate of opportunistic diseases and/or co morbidities. As a result, most of them also had longer ( $\geq 14$ days) hospital stay with unfavorable outcomes.

The overall prevalence of malnutrition was found to be high. WHO clinical stage 4 diseases and CD4 count $<200 / \mu \mathrm{L}$ were independently associated with malnutrition. We recommend comprehensive nutritional assessment and care with special emphasis on patients with CD4 count of less than $200 / \mu \mathrm{L}$ and/or those with advanced stages of the disease as part of their HAART care. We also recommend that large scale prospective cohort study be conducted including all HIV/AIDS patients in Ethiopian setup.

\section{REFERENCES}

1. Duggal S, Chugh T, Duggal A. HIV and Malnutrition: Effects on Immune System. Clinical and Developmental Immunology 2012: 8 .

2. Norman K, Pichard C, Lochs $\mathrm{H}$, et al. Prognostic impact of disease-related malnutrition. Clinical Nutrition,2005; 27: 515.

3. Hecker LM, Kotler DP. Malnutrition in patients with AIDS. Nutr Rev. 1990; 48(11): 393-401.

4. The Federal Democratic Republic of Ethiopia, Ministry of Health National Nutrition and HIV/AIDS Implementation Reference Manual, Ministry of Health Sept. 2008.

5. Brantley RK, Williams KR, Silva TM, et al. AIDS-associated diarrhea and wasting in Northeast Brazil is associated with subtherapeutic plasma levels of antiretroviral medications and with both bovine and human subtypes of Cryptosporidium parvum. Braz J Infect Dis. 2003; 7: 16-22.

6. Hailemariam S, Bune G, Ayele $\mathrm{H}$. Malnutrition: Prevalence and its associated factors in People living with HIV/AIDS, in Dilla University Referral Hospital. Arch Public Health, 2013; 71: 13.

7. Molla Daniel, Fekadu Mazengia, Dereje Birhanu. Nutritional status and associated factors among adult HIV/AIDS clients in Felege Hiwot Referral Hospital, Bahir Dar, Ethiopia. Science Journal of

Public Health, 2013; 1(1): 24-31.

8. Suttajit M. Advances in nutrition support for quality of life in HIV+/AIDS. Asia Pac J Clin Nutr, 2007;16 Suppl 1: 318-322.

9. Olalekan A Uthman et al. Prevalence and pattern of HIV-related malnutrition among women in sub-Saharan Africa: a meta-analysis of demographic health surveys. BMC Public Health, 2008, 8:226.

10. Noelle A. Benzekri, Jacques Sambou, Binetou Diaw, et al. High Prevalence of Severe Food Insecurity and Malnutrition among HIVInfected Adults in Senegal, West Africa. PLoS One. 2015 ; 10(11): e0141819.

11. WHO. Nutrient Requirements for People Living with HIV/AIDS: Report of a Technical Consultation, 2003).

12. Wen $\mathrm{H}$, Hua $\mathrm{J}$, Wei $\mathrm{C}$, et al. Malnutrition in hospitalized people living with HIV/AIDS: evidence from a cross-sectional study from Chengdu, China. Asia Pac J Clin Nutr, 2011; 20: 544-550.

13. Wanke CA, Silva M, Knox TA, et al. Weight loss and wasting remain common complications in individuals infected with human immunodeficiency virus in the era of highly active antiretroviral therapy. Clin Infect Dis, 2000; 31: 803-805.

14. Mehta SH, Astemborski J, Sterling TR, et al. Serum albumin as a prognostic indicator for HIV disease progression. AIDS Res Hum Retroviruses, 2006; 22: 14-21.

15. Paton NI, Sangeetha S, Earnest A, Bellamy R. The impact of malnutrition on survival and the CD4 count response in HIV-infected patients starting antiretroviral therapy. HIV Med,2006; 7: 323-330. 
16. Johannessen A, Naman E, Ngowi BJ, et al. Predictors of mortality in HIV-infected patients starting antiretroviral therapy in a rural hospital in Tanzania. BMC Infect Dis 2008; 8: 52.

17. Evans D, Maskew M, Sanne I. Increased risk of mortality and loss to follow-up among HIV-positive patients with oropharyngeal candidiasis and malnutrition before antiretroviral therapy initiation: a retrospective analysis from a large urban cohort in Johannesburg, South Africa. Oral Surg Oral Med Oral Pathol Oral Radiol, 2012; 113: 362-372.

18. Van der Sande, Marianne A., Van der Loeff et al. Body Mass Index at Time of HIV
Diagnosis: A Strong and Independent Predictor of Survival. Journal of Acquired Immune Deficiency Syndromes. 2004, 37(2): 1288-1294.

19. Bane A, Yohannes AG, Fekade D. Morbidity and mortality of adult patients with HIV/AIDS at Tikur Anbessa Teaching Hospital, Addis Ababa, Ethiopia. Ethiop Med J, 2003; 41: 131-140.

20. Meseret A, Tigist K, Negash B, et al. Prevalence of Anemia and Associated Risk Factors among Adult HIV Patients at the AntiRetroviral Therapy Clinic at the University of Gondar Hospital, Ethiopia. ScientificReports, 2013;2: 662. 